\title{
The use of elastic bandage and kinesiotherapy in lombar pain treatment: case report
}

\author{
Fernanda Cimão dos Santos'; Helen Katharine Christofel2; Ovídia Ignêz Pires²; Rodrigo Antonio Carvalho Andraus ${ }^{1,2}$.
}

\begin{abstract}
Background: Nowadays, lumbar pain may be one of the most frequent causes of medical leave, regardless of age and gender, and may have a known or unknown cause. Objective: To evaluate the existence of a decrease in pain and improvement in the lumbar functionalities using therapeutic elastic bandaging by the Therapy Taping ${ }^{\circledR}$ method and kinesiotherapy. Method: 23 years old subject, 80 pounds, 1.73 high, single, assists in the school transportation of children. The patient answered the questionnaires SF-36 Quality of Life Questionnaire, Oswetry 2.0 Disability Index, McGill pain questionnaire, Visual Analogue Scale and was submitted to Schöber's flexibility test, as well as an exercise protocol and elastic bandaging application in Region of pain for 6 weeks. Results: The results showed improvement in the tests, decrease of pain, gain of functionality and improvement in quality of life. Conclusion: The use of kinesiotherapy and elastic bandaging was effective for the treatment of chronic low back pain.
\end{abstract}

Keywords: Low Back Pain; Disability; Exercises.

\section{BACKGROUND}

Lumbar pain refers to all types of pain which occurs in the thoracolumbar, lumbar or lumbosacral region. It is one of the most common and frequent orthopedic diseases, causing functional incapacity in the subject and one of the reasons that causes more work leave and high costs in health care. More than $60-90 \%$ of the population has had an episode of acute low back pain (LBP) at least once in their lives and most of them are treated successfully, but between 5 and $15 \%$ of the population develop chronic LBP where there is no total relief of symptoms, which persist throughout life ${ }^{(1)}$. Lumbar pain is classified according to its duration, defined as acute if lasting from 2 to 4 weeks, subacute up to 12 weeks and chronic if it is more than 12 weeks. It is also classified in relation to its cause in specific LBP, where patients describe the pain as inconstant and diffuse, and non-specific cause where pain is described as consistent and accurate ${ }^{(2)}$. It is provoked by several factors the main ones are loss of segmental stabilization and muscular strength, decreased balance capacity and hypo-flexibility of both the lumbar and thoracic regions. With the increase of these structural manifestations, an increase in the adoption of abnormal postures may occur and, over time, result in an inability to perform normal activities on a day-to-day basis ${ }^{(3,4)}$. Persistent pain and reduced mobility result in disuse muscle atrophy and changes in the structure of the lumbar spine and surrounding tissues, resulting in strength deterioration of the abdominal and trunk muscles. Thus, the pathophysiological mechanisms of chronic LBP should be analyzed and understood in order to identify the most efficient therapy, in order that there is effectiveness in the treatment ${ }^{(5)}$. Kinesiotherapy is probably the most widely used intervention for the treatment of subjects with chronic nonspecific LBP. The exercise shows positive and low cost results and has been indicated in most of the clinical practice guidelines for chronic $\mathrm{LBP}^{(6)}$. A CORE and trunk stabilization training, segmental muscle gain, lumbar surgical mobility, and postural re-education and control programs are essential for effective treatment of chronic $L B P^{(7)}$. In addition to the aforementioned kinesiotherapy treatments, there is the option of using elastic bandaging therapy, it has been increasingly used in musculoskeletal conditions and sports injuries. One of the techniques used in its application is the Therapy Taping ${ }^{\circledR}$ method, which was created by physiotherapist Nelson Morini Junior and consists of the application of a thin, lightweight and elastic bandage with a layer of adhesive (Therapy Tex ${ }^{\circledR}$ ), elastic bandaging is used as a suggestion to send information afferent to the cerebral cortex, resulting in a better motor response ${ }^{(8)}$. The method based on the concept of inter tegumentary stimulation, on the principles of neurophysiology and part of the concept of elastic

Corresponding Author: Rodrigo Antonio Carvalho Andraus - Center for Research in Health Sciences - UNOPAR. Street Marseille $n{ }^{\circ}$. 491 , Jardim Piza. CEP: $86041-140$ Londrina, PR - Brazil. Phone: + 5543 3371-7990. E-mail: rodrigo.andraus@unopar.com.br

1 Faculdade de Apucarana (FAP), Apucarana (PR), Brazil.

${ }^{2}$ Master's Program in Rehabilitation Sciences, Universidade do Norte do Paraná (UNOPAR), Londrina (PR), Brazil.

Source of funding: None.

Submission date 15 May 2017; Acceptance date 02 October 2017; Publication date 02 April 2018 
deformation making the reactive force of the bandage provides the necessary information the musculoskeletal system, and this elastic tape guarantees free mobility of the segment. Though, bandaging promotes an elevation of the epidermis and reduces pressure on the mechanoreceptors which are located below the dermis, thereby reducing nociceptive stimuli. Other proposed benefits include improvement in blood and lymphatic circulation, reduction of pain intensity, realignment of the joints and change in the patterns of treated muscles of recruitment activity ${ }^{(8,9)}$. When the application is followed correctly, including the choice of the technique of application in or around the muscle as well as the knowledge of the pathology, there is evidence, mainly therapeutic, that the use of elastic bandages associated with other forms of intervention may be beneficial in the treatment of musculoskeletal disorders ${ }^{(10)}$. The purpose of the study was to examine the use of therapeutic elastic bandage by the Therapy Taping ${ }^{\circledR}$ method associated with kinesiotherapy in the treatment of chronic LBP.

\section{METHOD}

A case study, characterized as a descriptive study with an intentional sample, the ethical aspects were followed and approved by the Research Ethics Committee of the Apucarana College (protocol number: 1,625,652). This clinical case study carried out at the Physical Therapy Clinic in the Apucarana College. The evaluation and intervention performed in August and September 2016.

\section{Data collection instruments}

We selected a patient with chronic LBP of unknown origin who had not undergone any physiotherapeutic treatment in the last 3 months prior to the study. The subject was previously instructed and made aware of the research procedures and was asked to sign the Free and Informed Consent Form. In order to evaluate the patient, he underwent five evaluation procedures before the beginning and the end of treatment, namely: application of the SF-36 Quality of Life Questionnaire (SF-36), McGill Pain Questionnaire, Oswestry Index Evaluation 2.0 of Disability, Schöber's Flexibility Test and pain evaluation by Visual Analogue Scale (VAS). The SF-36 is a questionnaire composed of 36 questions that assess the conception of the disease from the perspective of the individual. The SF-36 was translated and validated in Brazil in 1997 by the researcher Rozana Mesquita Ciconelli, and it evaluates eight health concepts: functional capacity, physical aspect, pain, general health, vitality, social aspects, emotional aspects and mental health. The questionnaire was applied in a reserved room only in the presence of the interviewer and the research participant.

We also applied the McGill Pain Questionnaire, which characterizes and discerns the affective, sensory and evaluative components of pain through 78 words (descriptors) used to characterize the pain and its intensity variation. It is possible to obtain qualitative and quantitative information from verbal descriptions. The descriptors are organized into four categories (sensory, affective, evaluative and miscellaneous) and 20 subcategories. The pain index is reached by summing the intensity values of the chosen descriptors. The patient was further evaluated through the Oswestry 2.0 Disability Index, this is used for functional assessment of the subject encompassing measures of the effect of pain on the accomplishment of activities of daily living. The development of the Oswestry Disability Index was initiated by John O'Brien in 1976, and the first version was published only in 1980. It is a questionnaire consisting of 10 questions with 6 alternatives where each is scored on a hierarchical scale of 0 to 5 points. After the interpretation of the results, the individual's functional disability index is shown. Besides the questionnaires, the subject was submitted to the Schöber flexibility test, whose purpose is to measure the mobility of the lumbosacral segment. The test is performed with the patient in orthostatic position with the knees in extension and feet aligned and together. The examiner scratches a line with a dermatographic pencil at the height of the posterior superior iliac spines, and with a tape measure is measured $10 \mathrm{~cm}$ above the initial mark by scratching another line. The subject is then asked to perform a previous trunk flexion up to the limit supported. Then the examiner determines the distance between the two scratched lines by evaluating the flexibility of the segment. The patient was also submitted to VAS for pain assessment. It is a straight line, numbered 0 to 10 from one end to the other, 0 of which means total absence of pain and 10 the maximum level of

\section{CASE STUDY}

A female patient, 23 years old, $80 \mathrm{~kg}, 173 \mathrm{~cm}$ tall, single, student and school van driver. She lives in Apucarana, Paraná, Brazil. She reported that while she was working in January 2015 she felt severe back pain, however, she could not explain why. The pain was heightened by taking care of a close relative, who she moved incorrectly as a result of it she had severe pain in the lower back. The study participant claims that the pain was continuous and intensified by putting on weight and staying in position for an extended period of time, and at bedtime it was a mild pain relief. After the initial evaluation, the research participant underwent a total of 6 weeks of physiotherapeutic treatment, performed at a 3-times-a-week interval, consisting of lumbar pelvic mobility exercises, stretching and strengthening of the anterior and posterior trunk chain, CORE exercises and segmental (lumbar) stabilization. The application of elastic bandage by the Therapy Taping ${ }^{\circledR}$ method was performed twice weekly during the proposed 6 weeks of kinesiotherapy. The technique used was I with a fixed point at the level of the gluteal fold and movable point until the height of the last rib (bilateral) and a "cleavage" at the pain site, without a fixed point and without tension (Figure 1). 


\section{Chronological evolution of the intervention:}

On 08/08/2016, the SF-36, the Oswestry 2.0 Disability Index, the McGill Pain Questionnaire, the VAS and the Schöber Flexibility Test were applied. On August 10, 2016, the treatment was started with therapeutic exercises, performed three times a week, with sessions of approximately 60 minutes. The first application of elastic bandage by the Therapy Taping ${ }^{\circledR}$ method was performed on 08/11/2016, being applied twice a week. The patient used the bandage for 3 days and then it was removed. There were no changes in the exercise protocol during the intervention. The patient reported pain during the practice of the proposed exercises, but was able to perform them correctly from the beginning to the end of the study. From the 10th session, the subject reported did not have a specific point of pain, in which the bandage was placed by the "cleavage" technique. It is necessary to perform anterior trunk flexion and to palpate the spinal processes in search of some point of pain. There was a gradual improvement during the intervention protocol and from the 13th session the patient reported no more intense pain, only sporadic episodes of mild pain. The 18th session of kinesiotherapy and the last application of elastic bandage was on September $19^{\text {th }} 2016$. The evaluation tests performed at the start of treatment were applied again on $22^{\text {nd }}$ September 2016. Data analysis in the present study was descriptive.

\section{RESULTS}

In the SF-36, eight health concepts were evaluated in agreement with the patient's own vision. Values were obtained on a scale ranging from 0 to 100 , where zero is the worst state and one hundred is the best. There was an improvement in 7 items, except for the item limiting by emotional aspects that continued with the maximum value in both evaluations (Table 1).

Regarding the Schober's Test, the patient initially had a range of $15 \mathrm{~cm}$ before the intervention and $16 \mathrm{~cm}$ after the intervention. It was noted that the patient did not have a decrease in lumbar pelvic flexibility, nevertheless, after the reevaluation there was an increase of $1 \mathrm{~cm}$ in its flexibility compared to the previous finding. Figure 2 shows the results concerning the Oswestry Index of functional.

Table 1. Scores of the SF-36 quality of life questionnaire before and after intervention

\begin{tabular}{lcc}
\hline \multicolumn{1}{c}{ Health Concepts } & Before & After \\
\hline Functional capacity & 80 & 95 \\
Physical aspects limitation & 75 & 100 \\
Pain & 41 & 62 \\
General health status & 42 & 52 \\
Vitality & 45 & 75 \\
Social aspects & 50 & 100 \\
Limitation by emotional aspects & 100 & 100 \\
Mental health & 36 & 76 \\
\hline
\end{tabular}

It should be noted that the Oswestry Index assesses, through questions, the impairment of daily functional capacity caused by pain. A significant improvement in the functional capacity of the subject after the intervention protocol is evidenced, determining a $20 \%$ decrease in the patient's disability. The same was observed in the VAS evaluation of pain (Figure 3), which was in the pre-intervention period a pain chart 7 (moderate) and in the post-intervention period was 2 (mild), evidencing that there was a significant decrease in the patient's pain.

The results of the McGill Pain Questionnaire are presented in Figure 4, and it is verified there was a decrease in the number of descriptors chosen and variation of its intensity in $50 \%$ in total. Only in the affective item of the pain index there was an increase in relation to the characterization and intensity of the pain.

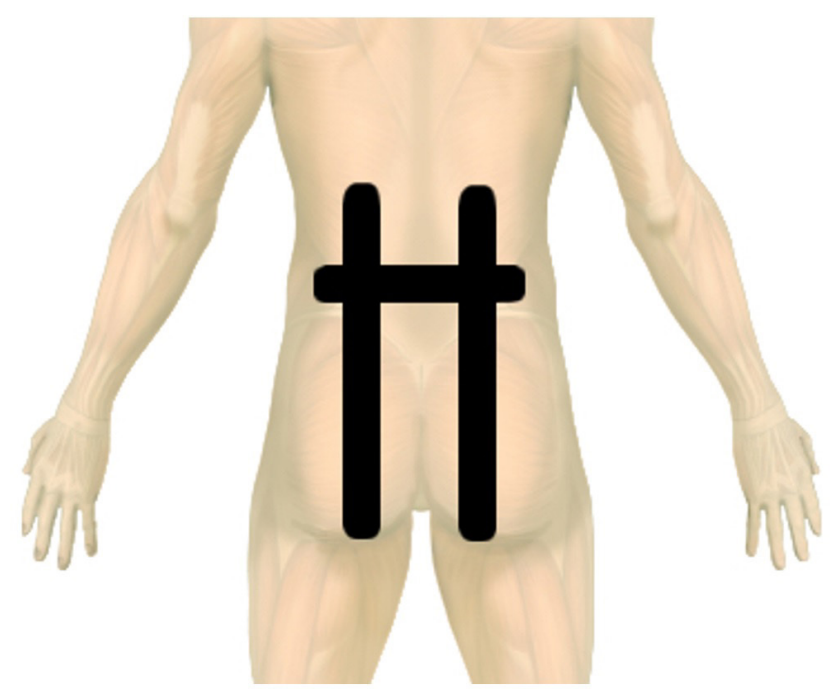

Figura 1. Illustrative image of the therapeutic elastic bandage applied by the Therapy Taping ${ }^{\circledR}$ method.

\section{Oswestry Disability Index (\%)}

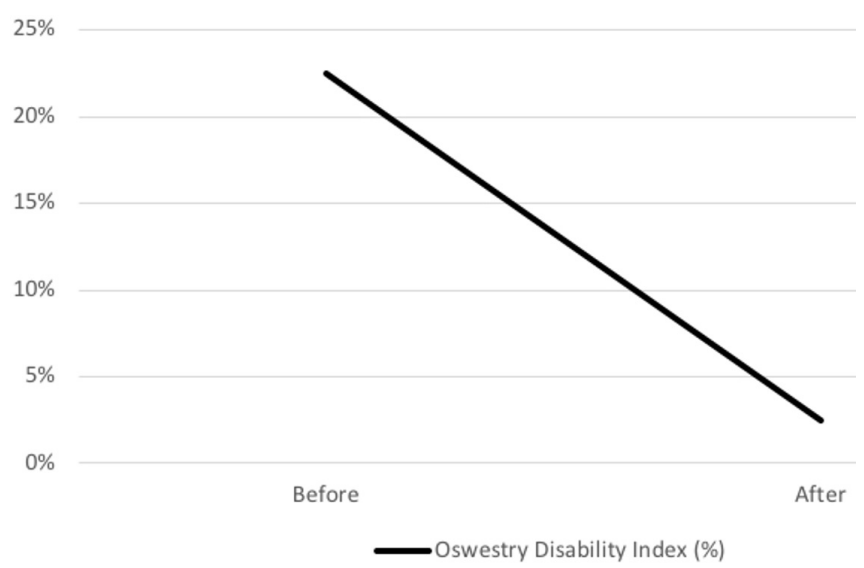

Figure 2. Results of the Oswestry Disability Index before and after the intervention protocol. 


\section{Visual Analog Scale for Pain (VAS)}

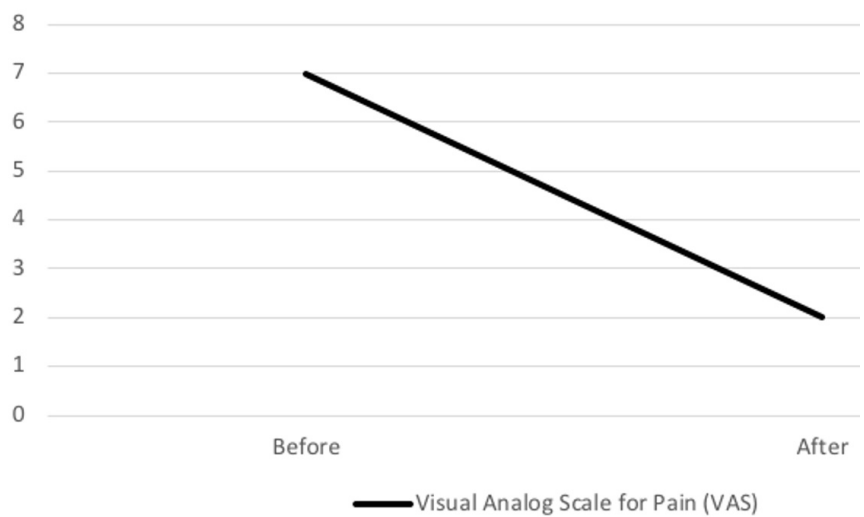

Figure 3. Visual Analog Scale for pain before and after the intervention protocol.

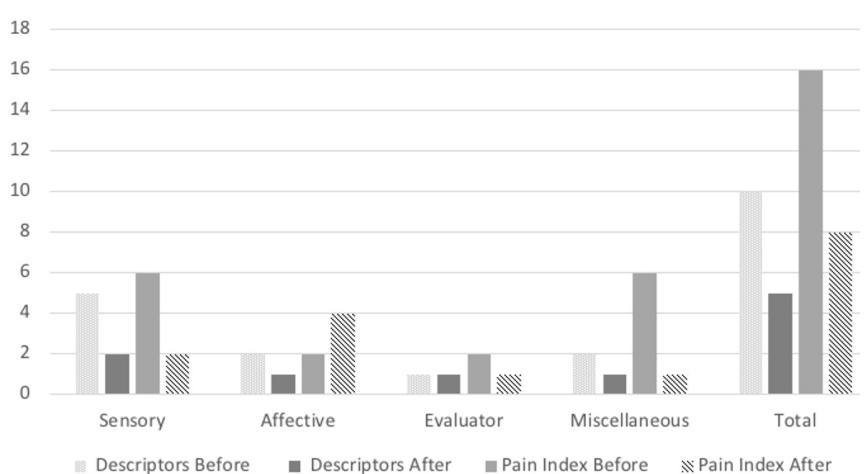

Figure 4. McGill Pain Questionnaire scores before and after the intervention.

\section{DISCUSSION}

The main risk factor for the onset of LBP is the weakness of the rectus abdominis, internal oblique, external oblique, transverse abdominal, spinal erector, lumbar square and, multifidus muscles, which constitute CORE. These muscles are mainly responsible for stabilizing the spine, as well as protecting and reducing the impact on the vertebrae and intervertebral discs. A strong CORE promotes segmental protection and stability, thus preventing acute and chronic pain with or without specific cause ${ }^{(11)}$. Conforming to Bae et al. (12), the application of an elastic bandage reduces the pain in patients with chronic LBP and consequently affects postural adjustments positively. Our findings corroborate with the aforementioned data, since there was a considerable improvement in the subject's functionality due to strength gain, stability and flexibility of the involved musculature, besides the attenuation of the segmental pain. For Parreira et al. ${ }^{(13)}$, although elastic bandage is widely used in clinical practice, it did not provide any statistically significant benefit in the treatment of musculoskeletal disorder pain, or its effect was too small to be clinically valid. Paoloni et al. (14) and Kachanathu et al. ${ }^{(15)}$ investigated the effect of the combination of exercises and elastic bandages in patients with chronic LBP and observed an improvement in the pain after the application of the bandage, however, a significant decrease in pain occurred after four weeks of treatment with therapeutic exercises in conjunction with elastic bandage. This corresponds to the present study, where the patient reported significant improvement only from the 13th session, which can be explained physiologically and there is a body adaptation time to the new routine of exercises and thus the muscular strength gain is gradual decrease, as well as a decrease in local pain. According Castro-Sánchez et al. ${ }^{(16)}$ and Kalron and Bar-Sela ${ }^{(10)}$, the elastic bandage can reduce pain for a short period of time after the application. The clinical value of the application of the bandage as a form is still small and does not support evidence that elastic bandaging has a positive effect on long-term pain treatments, as in the case of chronic pain. Compared to our findings, there was a significant decrease in pain, and when applied elastic bandage the patient reported a decrease in local pain after the application, providing an improvement in the functionality of daily life activities and support during exercise in the sessions, in addition to reducing or avoiding post-exercise pains. The decrease in chronic pain itself was attributed to the treatment of kinesiotherapy according to the therapeutic guidelines. There is insufficient information to indicate the use of elastic bandages as a single therapy for pain relief, but rather as adjunctive treatment to other interventions. In line with the literature, the quality that shows the use of elastic bandage is low. The relevance of this study is given to the clinical community, since this research shows positive results when compared to results of other authors. Thus, the study was limited to the analysis of a single case, and it is necessary to perform future comparative studies between the control group and the test group using the elastic bandage as a study.

\section{CONCLUSION}

The proposed Therapy Taping ${ }^{\circledR}$ elastic bandaging protocol associated with kinesiotherapy intervention proved to be effective in the treatment of chronic LBP, providing a significant reduction of pain, improvement of functional capacity in activities of daily living, improvement of flexibility of the lumbosacral region and consequently improving the quality of life of patient.

\section{AUTHOR'S CONTRIBUTION}

Each author contributed individually and significantly to the development of the manuscript. FCS, performed the data collection, followed the individual, gathered the clinical data and contributed to the drafting of the manuscript. HKC, conducted the manuscript review and contributed to the study's intellectual concept. OIP, contributed to the drafting of the manuscript. RACA, conducted the manuscript review and contributed to the study's intellectual concept.

\section{CONFLICTS OF INTEREST}

The authors declare no conflict of interest. 


\section{REFERENCES}

1. Byoung-Hwan OH, Hong-Hyun K, Cheol-Yong K, Chan-Woo N. Comparison of physical function according to the lumbar movement method of stabilizing a patient with chronic low back pain. Journal of Physical Therapy Science. 2015; 27(12): 3655-3658.

2. Compare A, Marchettini P, Zarbo C. Risk Factors Linked to Psychological Distress, Productivity Losses, and Sick Leave in Low-Back-Pain Employees: A Three-Year Longitudinal Cohort Study. Pain Research and Treatment. 2016; 1-9.

3. Min-Yeong $\mathrm{H}$, Kyoung $\mathrm{K}$, Beom-Young $\mathrm{H}$, Chan-Woo $\mathrm{N}$. The effect of lumbar stabilization exercises and thoracic mobilization and exercises on chronic low back pain patients. Journal of Physical Therapy Science. 2015; 27(12):3843-3846.

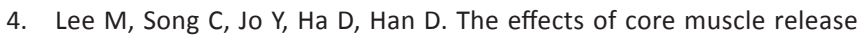
technique on lumbar spine deformation and low back pain. Journal of Physical Therapy Science. 2015; 27(5):1519-22.

5. Massé-Alarie H, Schneider C. Revisiting the Corticomotor Plasticity in Low Back Pain: Challenges and Perspectives. Healthcare. 2016; 4(3): 67.

6. Yamato TP, Maher CG, Saragiotto BT, Hancock MJ, Ostelo RW, Cabral CM, Menezes Costa LC, Costa LO. Pilates for low back pain. Cochrane Database of Systematic Reviews. 2015; 2(7):CD010265.

7. Javadian Y, Akbari M, Talebi G, Taghipour-Darzi M, Janmohammadi N. Influence of core stability exercise on lumbar vertebral instability in patients presented with chronic low back pain: a randomized clinical trial. 2015; 6(2): 98-102.

8. Morini Jr, N. Bandagem Terapêutica: conceito de estimulação tegumentar. São Paulo: Roca; 2015.
9. Lemos TV, Albino AC, Matheus JP, Barbosa Ade M. The Effect of Kinesio Taping in Forward Bending of the Lumbar Spine. Journal of Physical Therapy Science. 2014; 26(9):1371-5.

10. Kalron A, Bar-Sela S. A systematic review of the effectiveness of Kinesio Taping - fact or fashion? European Journal Physical and Rehabilitation Medicine. 2013; 49(5):699-709.

11. Hwi-young C, Eun-hye K, Junesun K. Effects of the CORE Exercise Program on Pain and Active Range of Motion in Patients with Chronic Low Back Pain. Journal of Physical Therapy Science. 2014; 26(8): 1237-1240.

12. Bae SH, Lee JH, Oh KA, Kim KY. The Effects of Kinesio Taping on Potential in Chronic Low Back Pain Patients Anticipatory Postural Control and Cerebral Cortex. Journal of Physical Therapy Science. 2013; 25(11): 1367-1371.

13. Parreira PC, Costa LC, Hespanhol LC Jr, Lopes AD, Costa LO. Current evidence does not support the use of Kinesio Taping in clinical practice: a systematic review. Journal of Physiotherapy. 2014; 60(1):31-9.

14. Paoloni M, Bernetti A, Fratocchi G, Mangone M, Parrinello L, Del Pilar Cooper $\mathrm{M}$, et al. Kinesio Taping applied to lumbar muscles influences clinical and electromyographic characteristics in chronic low back pain patients. European Journal Physical and Rehabilitation Medicine. 2011; 47(2):237-44.

15. Kachanathu SJ, Alenazi AM, Seif HE, Hafez AR, Alroumim MA. Comparison between Kinesio Taping and a Traditional Physical Therapy Program in Treatment of Nonspecific Low Back Pain. Journal of Physical Therapy Science. 2014; 26(8): 1185-1188.

16. Castro-Sánchez AM, Lara-Palomo IC, Matarán-Peñarrocha GA, FernándezSánchez M, Sánchez-Labraca N, Arroyo-Morales M. Kinesio Taping reduces disability and pain slightly in chronic non-specific low back pain: a randomised trial. Journal of Physiotherapy. 2012; 58(2):89-95. 